\title{
Extraction Optimization of Polysaccharides From Corn Silk and Their Antioxidant Activities in vitro and in vivo
}

\author{
Liang Zhang, Yang Yang and Zhanyong Wang* \\ College of Bioscience and Biotechnology, Shenyang Agricultural University, Shenyang, China
}

Response surface technique was employed for improving the extraction of corn silk polysaccharides (CSP). Temperature, liquid-to-solid ratio, and per extraction time were all examined as separate factors. The optimal extraction parameters were determined by fitting experimental data to a second-order polynomial; a liquid-to-solid ratio of $21.5 \mathrm{ml} / \mathrm{g}$, temperature equivalent to $88^{\circ} \mathrm{C}$, and extraction time of $1.87 \mathrm{~h}$. The experimental yield of the extracted polysaccharides following the application of these conditions was $4.33 \pm 0.08 \%$ (dry weight), which fit quite well with the predicted value. CSP's strong scavenging

OPEN ACCESS

Edited by:

Haibin Tong,

Wenzhou University, China

Reviewed by:

Dianhui Luo,

Huaqiao University, China

Jianhua Liu,

Zhejiang University of Technology,

China

*Correspondence:

Zhanyong Wang

wangzy125@gmail.com

Specialty section:

This article was submitted to

Ethnopharmacology,

a section of the journal

Frontiers in Pharmacology

Received: 08 July 2021

Accepted: 18 August 2021

Published: 08 September 2021

Citation:

Zhang L, Yang Y and Wang Z (2021)

Extraction Optimization of

Polysaccharides From Corn Silk and

Their Antioxidant Activities in vitro and

in vivo.

Front. Pharmacol. 12:738150.

doi: $10.3389 /$ fphar.2021.738150 capabilities against hydroxyls, 1,1-diphenyl-2-picrylhydrazyl radicals, and superoxide anions along with its excellent reducing potential, were demonstrated in an in vitro antioxidant experiment. Meanwhile, in vivo testing revealed that CSP substantially enhanced glutathione peroxidase and superoxide dismutase activities. The Malondialdehyde levels in the liver and serum of aged mice also underwent a decrease. This study found that CSP has a substantial antioxidant potential in vitro and in vivo, suggesting that it might be used as an antioxidant in food and medicine.

Keywords: polysaccharide, antioxidant activity, extraction, anti-aging, corn silk

\section{INTRODUCTION}

With the improvement of lifestyle, people are becoming more aware of their health. Hence, the demand for health care products has manifested a noticeable rise. However, people do not only consider the efficacy, but also the source of these health care products, showing specific preference to natural health care products that are obtained from animals or plants (Necyk and Zubach-Cassano, 2017). For instance, some natural health care products are commonly used as antioxidants. Antioxidants can scavenge reactive oxygen species, which can cause oxidative stress-related illnesses (Das and Roychoudhury, 2014). Even though synthetic antioxidants can prevent or minimize the damage caused by reactive oxygen species, the demand for natural antioxidants, particularly those derived from plants, has risen in recent years owing to the former's possible toxicological consequences (Ge et al., 2013; Shah et al., 2014). In recent years, natural polysaccharides with antioxidant activity have attracted increasing attention in natural medicine research and production (Yu et al., 2018). Corn silk is formed from stigmas, which are the yellowish thread-like strands that come from the female maize flower. It is a waste product from maize production, however, is quite plentiful (Hasanudin et al., 2012). Corn silk is a famous traditional herbal drug. In China, people have been using corn silk decoction for diabetes therapy for decades (Zhao et al., 2012). It is also used as traditional medicine in Turkey, France, and the United States (Hasanudin et al., 2012). 
Carbohydrates, flavonoids, alkaloids, steroids, saponins, volatile oils, tannins, vitamins, and proteins are among the bioactive components of maize silk that have been frequently documented (Liu et al., 2011; Zhao et al., 2017). In particular, the polysaccharides extracted from corn silk (CSP) reportedly exhibited anti-fatigue (Zhao et al., 2017), anti-obesity (Chaiittianan et al., 2016), anti-diabetic effects (Zhao et al., 2012; Pan et al., 2017), antioxidant activity (Chen et al., 2013), and anti-hepatoma (Yang et al., 2014) activities.

RSM (response surface methodology) refers to a statistical strategy for the optimization of complicated operations. RSM's major benefit is that it requires fewer experimental trials to examine various factors and their interactions (Bezerra et al., 2008). In the realm of polysaccharide extraction, RSM is widely employed (Li et al., 2017; Raza et al., 2017; Wu et al., 2017). The goal of the current investigation was to use RSM to optimize the influence of extraction temperature, per extraction duration, and liquidto-solid ratio on CSP aqueous extraction yield and to assess the antioxidant properties of CSP. This research sheds light on how CSP can be employed as a natural antioxidant in the pharmaceutical and food sectors.

\section{MATERIALS AND METHODS}

\section{Chemicals and Materials}

Corn silk was gathered in the Fushun suburbs, Liaoning Province, China. Sigma Chemicals Co. (St. Louis, MO, United States) provided ascorbic acid, D-galactose (D-gal), and 1,1-diphenyl2-picrylhydrazyl (DPPH) and Sinopharm Chemical Regent Co., Ltd., Shanghai, China, furnished the rest of the reagents and solvents. All compounds used were analytical grade unless otherwise specified.

\section{Extraction of NCP}

Corn silk was crushed and defatted in an ethanol bath at $80^{\circ} \mathrm{C}$. The defatted sample $(10 \mathrm{~g})$ was extracted using deionized water at a certain extraction temperature, duration, and liquid-to-solid ratio after vacuum drying. The mixture was subjected to centrifugation for $15 \mathrm{~min}$ at $4,000 \mathrm{r} / \mathrm{min}$ to obtain the supernatant after extraction. The supernatant was combined with three portions of ethanol and stored at $4{ }^{\circ} \mathrm{C}$ for $24 \mathrm{~h}$ before being centrifuged again to obtain the precipitate. Centrifugation $(2000 \times \mathrm{g}, 15 \mathrm{~min})$ was used to extract the polysaccharide precipitate, which was subsequently deproteinated by employing a combination of proteinase and the Sevag technique (Gao et al., 2013). The CSP was obtained by lyophilizing the supernatant, which was then lyophilized to powder. The phenol-sulfuric acid technique was utilized to determine the polysaccharide content of the specimen, with D-glucose as the reference (Dubois et al., 1956).

\section{Design of the Experiment}

The initial range of variables was defined for the extraction process and then a Box-Behnken design (BBD) was uptaken for testing the response (Y, Yield) against three independent variables: temperature (X1), liquid-to-solid ratio (X2), and per extraction time (X3). The design of the experiments was carried out using a $\mathrm{BBD}$, at three levels with three factors and individual independent variables coded at three levels ranging from $-1,0$, and +1 . The variables were coded following Eq. 1 for statistical calculations:

$$
x_{i}=\frac{X_{i}-X_{0}}{\Delta X},
$$

Where $x_{i}$ denotes a dimensionless value of an independent variable, $x_{0}$ refers to the actual magnitude of an independent variable at the center point, $x_{i}$ stands for the actual magnitude of an independent variable, and $\Delta x$ denotes the step change of the actual magnitude of the variable $i$ corresponding to a variation of a unit for its dimensionless value.

The design, as indicated in Table 1, comprises 17 experimental locations with five center points to allow for pure error estimates. Each trial's answer value was the average of duplicates.

To fit the second-order polynomial to the experimental data and for identification of the key components of the model, a nonlinear regression approach was utilized. We described the quadratic response model by taking into account all of the linear, square, and interaction components as

$$
\mathrm{Y}=A_{0}+\sum_{i=3}^{3} A_{i} X_{i}+\sum_{i=3}^{3} A_{i i} X_{i}^{2}+\sum \sum_{i<j=1}^{3} A_{i j} X_{i} X_{j},
$$

Where $A_{O}$ stands for a constant, and $A_{i}, A_{i i}$, and $A_{i j}$ refer to the respective coefficients of the linear, quadratic, and interactive terms, $X_{i}$ and $X_{j}$ represent the levels of the independent variables. To illustrate the link among the experimental and response levels of individual elements and to find the optimal circumstances, the fitted polynomial expression is profiled into surface and contour plots (Wang et al., 2015).

The experimental data were subjected to analysis by employing Design-Expert (Version 11.0.3, Stat-Ease Inc., United States) software. Three further experiments were carried out after that to ensure that the statistical experimental techniques were valid.

\section{Antioxidant Activity Assay in vitro}

Hydroxyl radical scavenging activity: The potential of CSP for scavenging hydroxyl radicals was tested by making use of the method published by Smirnoff and Cumbes (1989), with minor changes. $1 \mathrm{ml}$ of $8.8 \mathrm{mmol} / \mathrm{L} \mathrm{H}_{2} \mathrm{O}_{2}, 1 \mathrm{ml}$ of $9 \mathrm{mmol} / \mathrm{L}$ salicylic acid-ethanol, $1 \mathrm{ml}$ of $9 \mathrm{mmol} / \mathrm{L} \mathrm{FeSO}_{4}$, and $1 \mathrm{ml}$ of the sample solution was used in the reaction mixture. The absorbance of the combination was quantified at $510 \mathrm{~nm}$ following $30 \mathrm{~min}$ of incubation at $37^{\circ} \mathrm{C}$ with the positive control as ascorbic acid. The \% hydroxyl radical scavenging activity was quantified making use of the following expression:

Hydroxyl radical scavenging activity $(\%)=\left(1-\frac{A_{s}-A_{s 0}}{A_{c}}\right) \times 100$,

Where $A_{s}$ is the absorbance of the test sample, $A_{c}$ denotes the absorbance of the control (deionized water instead of sample 
TABLE 1 | Box-Behnken design and results for extracted yield of CSP.

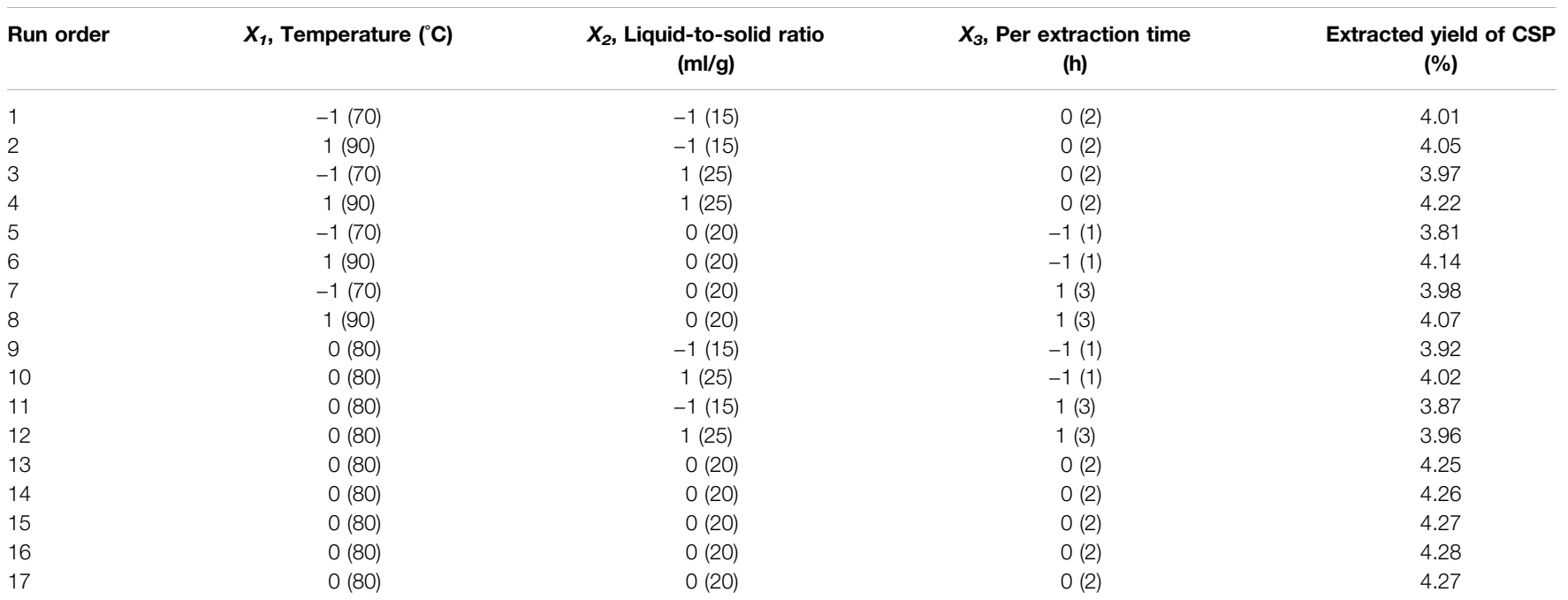

solution), and $A_{s 0}$ refers to the absorbance of the sample only (deionized water in place of $\mathrm{H}_{2} \mathrm{O}_{2}$ solution).

Superoxide radical scavenging activity: The scavenging ability of CSP against superoxide anion radicals was measured by employing the technique published by Tian et al. (2017). The $0.4 \mathrm{ml}$ sample solution was combined with Tris- $\mathrm{HCl}$ buffer $(\mathrm{pH}$ $8.2,50 \mathrm{mmol} / \mathrm{L}, 4.5 \mathrm{ml}$ ) and allowed to incubate at $25^{\circ} \mathrm{C}$ for $20 \mathrm{~min}$. After the addition of $0.1 \mathrm{ml}$ of $2.5 \mathrm{mmol} / \mathrm{L}$ pyrogallol solution to the mixture, it was set to incubate at $25^{\circ} \mathrm{C}$ for $5 \mathrm{~min}$. The addition of $0.1 \mathrm{ml}$ of $10 \mathrm{~mol} / \mathrm{L} \mathrm{HCl}$ to the mixture resulted in the termination of the reaction. The reaction mixture's absorbance was quantified at $325 \mathrm{~nm}$. The equation outlined below was utilized for ascertaining the superoxide radical scavenging activity:

$$
\text { Superoxide radical scavenging activity }(\%)=\left(1-\frac{A_{s}}{A_{c}}\right) \times 100 \text {, }
$$

Where $A_{s}$ refers to the absorbance of the samples, $A_{c}$ represents the absorbance of the control (deionized water instead of the sample).

DPPH radical scavenging activity: The assessment of DPPH radical scavenging activity was performed using Chaiklahan et al. (2013) modified technique (2013). A combination of $1 \mathrm{ml}$ sample and $2 \mathrm{ml} 0.1 \mathrm{~mol} / \mathrm{L} \mathrm{DPPH}$ was set to incubate in the dark at $25^{\circ} \mathrm{C}$ for $30 \mathrm{~min}$. The mixture's absorbance was estimated at $517 \mathrm{~nm}$. The equation given below was employed for the calculation of the potential to scavenge the $\mathrm{DPPH}$ radical:

$$
D P P H \text { radical scavenging activity }(\%)=\left(1-\frac{A_{s}}{A_{c}}\right) \times 100,
$$

Where $A_{s}$ represents the absorbance of the specimen, $A_{c}$ refers to the absorbance of the control (deionized water instead of the specimen).

$\mathrm{Fe} 3+$ reducing antioxidant power (FRAP): FRAP was calculated using the technique given by Yildirim et al. (2001) with minor changes. $2.5 \mathrm{ml}$ of $0.2 \mathrm{M}$ phosphate buffer ( $\mathrm{pH} 6.8$ ), $2.5 \mathrm{ml}$ of $1 \%(\mathrm{w} / \mathrm{v})$ potassium ferricyanide, and $2 \mathrm{ml}$ of polysaccharide sample solution made up the reaction mixture. After $30 \mathrm{~min}$ at $50^{\circ} \mathrm{C}$, the mixture was treated with $2.5 \mathrm{ml}$ of trichloroacetic acid $(10 \% \mathrm{w} / \mathrm{v})$ to stop the reaction and then placed into a centrifuge for $10 \mathrm{~min}$ at $4,000 \mathrm{r} / \mathrm{min}$. Finally, $2.5 \mathrm{ml}$ of the supernatant was added to $2.5 \mathrm{ml}$ of deionized water and $0.5 \mathrm{ml}$ of ferric chloride $(0.1 \% \mathrm{w} / \mathrm{v})$ in $2.5 \mathrm{ml}$ of deionized water. At $700 \mathrm{~nm}$, the absorbance was measured.

\section{Antioxidant Activity Assay in vivo}

The studies were conducted with 60 Kunming mice (2-months of age, body mass BW $=20 \pm 2 \mathrm{~g}$ ), with males and females in equal numbers. The mice were acquired from Jilin University's Pharmacology Experimental Center (Changchun, China). For 7 days, the mice were kept at a constant temperature of $25^{\circ} \mathrm{C}$, humidity of $55 \%$, and the light cycle of $12 \mathrm{~h}$ of light/12 h of darkness. All mice were given uninterrupted access to a normal laboratory pellet meal and water during the experiment (including acclimatization). All operations were performed by strictly adhering to the Chinese laws governing the utilization and treatment of laboratory animals.

Random assignment of the mice was made into six groups $(\mathrm{n}=$ 10 , equal number of males and females) following a 7 day acclimation period: normal control group, D-Gal group (aging control group), ascorbic acid group (100 mg/kg BW, as positive control), and CSP groups $(100,200$, and $400 \mathrm{mg} / \mathrm{kg} \mathrm{BW}$, as positive control). The D-Gal group received D-Gal $(100 \mathrm{mg} / \mathrm{kg}$ BW) by hypodermic injection while the normal control group received an identical amount of physiological saline via gastric gavage once daily. D-Gal (100 mg/kg BW) by hypodermic injection and ascorbic acid (100 mg/kg BW) by gastric gavage was given to the ascorbic acid group once daily. CSP (as doses of 100,200 , and $400 \mathrm{mg} / \mathrm{kg} \mathrm{BW}$ per day) and D-Gal (100 mg/kg BW per day) were given to the CSP groups through gastric gavage and hypodermic injection, respectively. For 40 days, all of the groups were fed once a day. 
TABLE 2 | Sequential model fitting for the yield of CSP.

\begin{tabular}{lccccc}
\hline \multirow{2}{*}{ Source } & \multicolumn{5}{c}{ Statistical analysis } \\
\cline { 2 - 6 } & Sum of Squares & $\boldsymbol{d} \boldsymbol{c}$ & Mean Square & $\boldsymbol{F}$-Value & $\boldsymbol{p}$-value \\
\hline Model & 0.3767 & 9 & 0.0419 & 34.09 & $<0.0001$ \\
$X_{1}$ & 0.0630 & 1 & 0.0630 & 51.32 & 0.0002 \\
$X_{2}$ & 0.0128 & 1 & 0.0128 & 10.42 & 0.0145 \\
$X_{3}$ & 0.0000 & 1 & 0.0000 & 0.0102 & 0.9225 \\
$X_{1} X_{2}$ & 0.0110 & 1 & 0.0110 & 8.98 & 0.0200 \\
$X_{1} X_{3}$ & 0.0144 & 1 & 0.0144 & 11.73 & 0.0111 \\
$X_{2} X_{3}$ & 0.0000 & 1 & 0.0000 & 0.0204 & 0.8906 \\
$X_{1}{ }^{2}$ & 0.0224 & 1 & 0.0224 & 18.27 & 0.0037 \\
$X_{2}{ }^{2}$ & 0.0717 & 1 & 0.0717 & 58.40 & 0.0001 \\
$X_{3}{ }^{2}$ & 0.1568 & 1 & 0.1568 & 127.73 & $<0.0001$ \\
Residual & 0.0086 & 7 & 0.0012 & - & - \\
Lack of Fit & 0.0081 & 3 & 0.0027 & 20.71 & 0.0067 \\
Pure Error & 0.0005 & 4 & 0.0001 & - & - \\
Cor Total & 0.3853 & 16 & - & - & - \\
& & & & &
\end{tabular}

The mice were weighed and decapitated $24 \mathrm{~h}$ after the last medication treatment. To extract serum, blood specimens were taken and centrifuged at $4,000 \times \mathrm{g}$ for $10 \mathrm{~min}$ at $4^{\circ} \mathrm{C}$. The liver was removed, weighed, and homogenized in $0.1 \mathrm{~g} / \mathrm{ml}$ wet weight icecold physiological saline right away. The supernatant was recovered after centrifuging the suspension. The entire set of treatments was conducted at a temperature of $4 \mathrm{C}$.

By making use of the kits obtained from Nanjing Jiancheng Bioengineering Institute (Nanjing, China), total antioxidant capacity (TAOC), and the levels of glutathione peroxidase (GSH-Px), malondialdehyde (MDA), and superoxide dismutase (SOD) in the supernatant and blood of the liver were determined The Modified BCA Protein Assay Kit was procured from Shanghai Sangon Biological Engineering Technology and Services Co., Ltd. (Shanghai, China) to determine the protein content.

\section{Statistical Analysis}

For statistical data interpretation, the Student's t-test was employed. The entire set of data within the text as well as the graphics is represented as mean \pm standard deviation unless otherwise specified. The computations were performed using SPSS (version 13.0).

\section{RESULTS AND DISCUSSION}

\section{BBD Analysis and Model Fitting}

We used BBD to run experiments with various combinations of physical characteristics to assess the combined influence of independent factors on the response. Table 1 shows the outcomes of these trials. Under the following experimental circumstances, the highest NCP yield was recorded: extraction temperature of $80^{\circ} \mathrm{C}$, the liquid-to-solid ratio of 20 , and per extraction duration of $2 \mathrm{~h} .4 .28 \%$ was the highest NCP yield. Between the experimental findings, an empirical connection described by a second-order polynomial equation with interaction terms was fitted. The following is the equation derived in terms of coded factors:

$$
\begin{aligned}
\mathrm{Y}= & 4.27+0.0887 X_{1}+0.04 X_{2}-0.0012 X_{3}+0.0525 X_{1} X_{2} \\
& -0.06 X_{1} X_{3}-0.0025 X_{2} X_{3}-0.073 X_{1}^{2}-0.1305 X_{2}^{2}-0.193 X_{3}^{2} .
\end{aligned}
$$

The regression coefficients were analyzing for their significance by observing their associated $p$-values, and the experimental data were examined using Pareto ANOVA (Table 2). The linear coefficients (X1 and X2), quadratic term coefficients (X12, X22, and X32), and interaction term coefficients (X1 X2 and X1 X3) were all significant $(p<0.05)$ according to the $p$-values of each model term in Table 2. The model was significant with an F-value of 34.09 and a $p$-value $<0.0001$. Due to relative pure error, the lack of fit F-value of 20.71 and related $p$-value of 0.0067 was not significant. The quadratic regression model's coefficient of determination $\left(R^{2}=0.9777\right)$ revealed that the model only explained $0.0223 \%$ of the overall changes. The adjusted coefficient of determination $\left(R^{2} a d j=0.949\right)$ is quite high, indicating that the model was significant, and the coefficient of variation (C.V. $=8.59 \%)$ clearly demonstrated that the experimental results were precise and reliable. Within the spectrum of experimental variables, the model was deemed adequate for prediction.

\section{Optimization and Verification of the Model}

To demonstrate the primary and interaction impacts of independent factors on a response variable, three-dimensional response surfaces and contour plots (Figure 1) were created. For two test variables, each curve represented an unlimited number of possibilities, while the other component was set to zero.

When the extraction duration (X3) was set to 0 , the temperature (X1) and liquid-to-solid ratio (X2) had quadratic impacts on the yields, as illustrated in Figures 1A,D. The yield was calculated at various temperatures and liquid-to-solid ratios while the extraction time was kept constant. When the temperature and liquid-to-solid ratios were $88^{\circ} \mathrm{C}$ and $21.5 \mathrm{ml} /$ g, respectively, the highest yield was obtained. Figures 1B,E depict the $3 \mathrm{D}$ response surface and contour plots at various temperatures (X1) and extraction times (X3) with a constant liquid-to-solid ratio (X2). When the extraction period was raised from 1 to $1.87 \mathrm{~h}$, the yield rose, but it dropped after that. The yield rose when the temperature was incremented from $70^{\circ} \mathrm{C}$ to $88^{\circ} \mathrm{C}$ but did not exceed beyond $88^{\circ} \mathrm{C}$. Figures 1C,F indicate that while the temperature (X1) was kept constant at level 0 , the liquid-tosolid ratio (X2) and per extraction time (X3) had quadratic impacts on the response yield. As the liquid-to-solid ratio underwent an increase and the pre-extraction time also demonstrated an increase, there was first an increase in the yield which dropped later. When the liquid-to-solid ratio and per extraction time were $21.5 \mathrm{ml} / \mathrm{g}$ and $1.87 \mathrm{~h}$, respectively, the highest yield was obtained.

The anticipated yield values (4.31\%) were reached under the following conditions: temperature of $88^{\circ} \mathrm{C}$, the liquid-to-solid ratio of $21.5 \mathrm{ml} / \mathrm{g}$, and per extraction time of $1.87 \mathrm{~h}$, according to the plots. The verified experimental yield was $4.33 \pm 0.08 \%$ at the above optimum circumstances. The mean value calculated from 

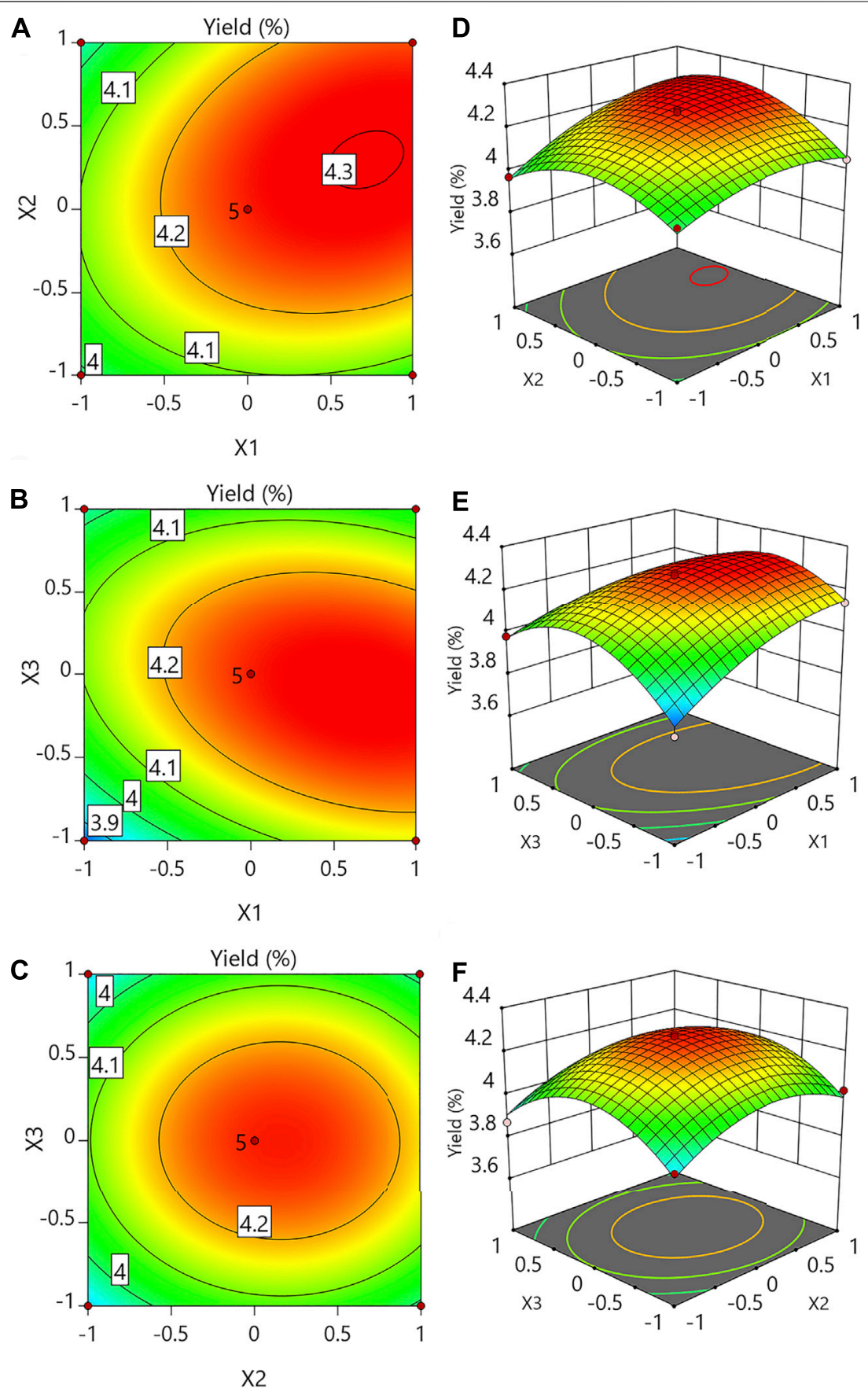

FIGURE 1 |Contour plots $(\mathbf{A}-\mathbf{C})$ and response surface plots (D-F) showing the effects of variables $\left(X_{1}\right.$ : temperature; $X_{2}$ : liquid-to-solid ratio; $X_{3}$ : per extraction time) on the response $Y$ (yield of CSP).

real trials verified the RSM model and suggested that it was acceptable for extraction when compared to the projected value.

\section{Antioxidant Activities of CSP in vitro}

The Fenton reaction or the iron-catalyzed Haber-Weiss reaction create hydroxyl radicals. They are so reactive that they can harm most biomolecules, although antioxidants can minimize their effects ( $\mathrm{Li}$ et al., 2012). Figure 2 depicts that the scavenging influence of CSP on hydroxyl radicals increased across the concentration range examined, but the scavenging effect of ascorbic acid on hydroxyl radicals was significantly greater $(p<0.05)$. CSP's $\mathrm{EC}_{50}$ against hydroxyl radicals was $918.2 \mathrm{mg} /$ $\mathrm{L}$, which was much greater than ascorbic acid's (365.6 mg/L). CSP has an $\mathrm{EC}_{50}$ similar to EUPS-2 (CSP extracted using an enzymolysis-ultrasonic technique) (Chen et al., 2014). There are two antioxidant processes for hydroxyl radicals: inhibition 


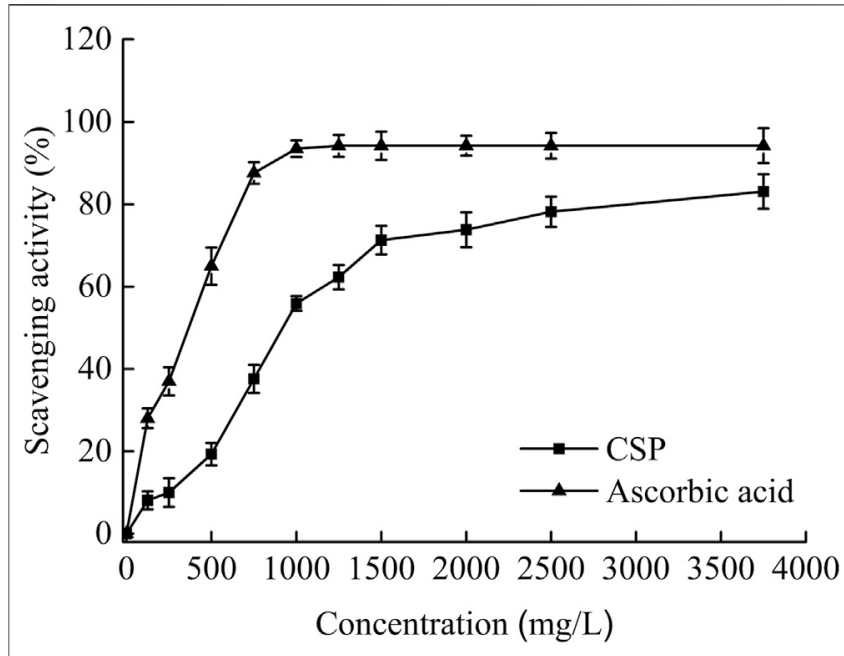

FIGURE 2 | Scavenging effects on hydroxyl radicals of CSP.

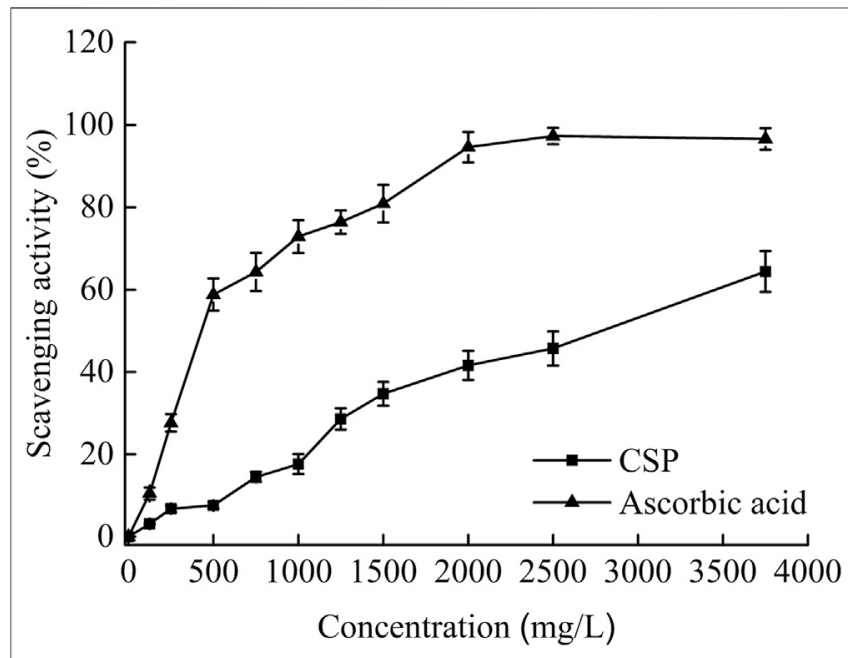

FIGURE 3 | Scavenging effects on superoxide anion radicals of CSP.

of hydroxyl radical production and direct scavenging of hydroxyl radicals (Ueda et al., 1996). The interaction of hydrogen with radicals and the subsequent cessation of the radical chain reaction was also described as part of the hydroxyl radical scavenging process by polysaccharides (Shi et al., 2013). As a result, the mechanism by which CSP scavenges hydroxyl radicals has to be investigated further.

A specific number of superoxide anion radicals are found within the human body. When superoxide anion radicals mix with hydroxyl radicals, cell DNA is damaged, and the human body's function is compromised (Tian et al., 2017). Figure 3 indicates that when the concentration of CSP rose, the rate of elimination of superoxide anion radicals increased, and the scavenging rate was proportional to the CSP concentration within the test dose range. The $\mathrm{R}^{2}$ coefficient was 0.9867 . Within the test dose range, CSP scavenging rates were lower

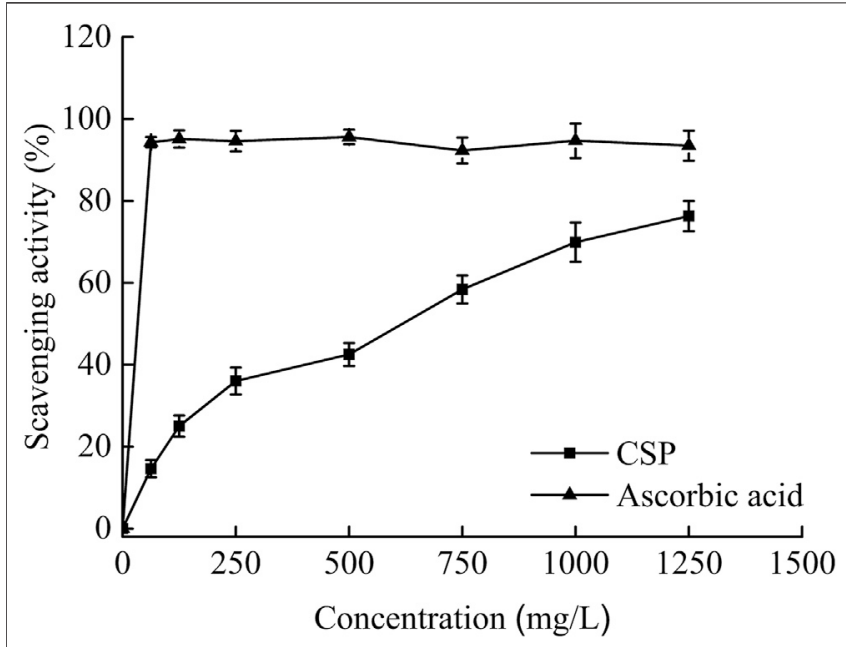

FIGURE 4 | Scavenging effects on DPPH radicals of CSP.

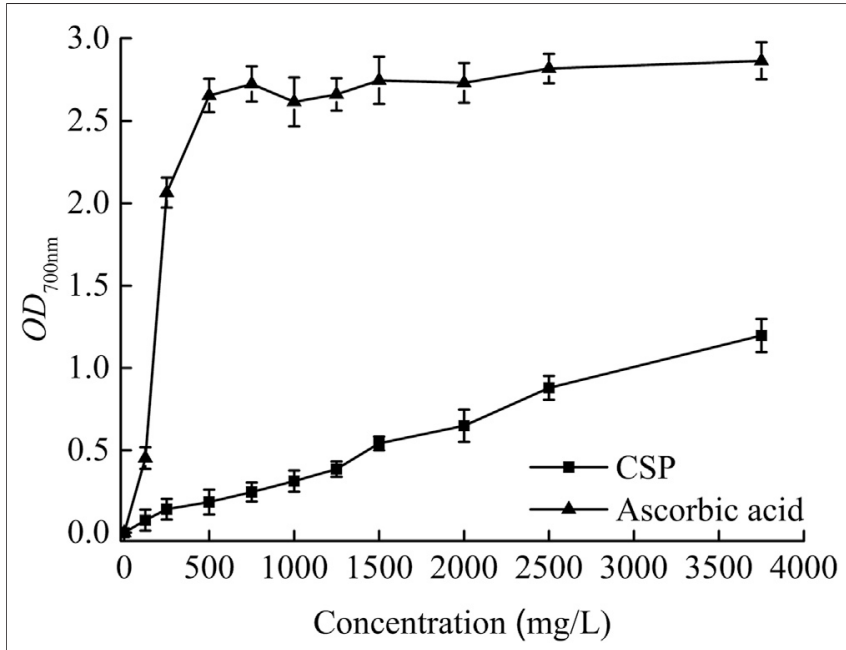

FIGURE 5 | $\mathrm{Fe}^{3+}$ reducing antioxidant power of CSP.

than ascorbic acid scavenging rates. CSP had a greater $\mathrm{EC}_{50}$ $(2,775.6 \mathrm{mg} / \mathrm{L})$ than ascorbic acid $(402.3 \mathrm{mg} / \mathrm{L})$. Although superoxide is a mild oxidant, it can deteriorate over time and produce additional reactive oxygen entities for instance singlet oxygen and hydroxyl radicals, which can lead to lipid peroxidation (Qi et al., 2005). Pathological events, such as arthritis and Alzheimer's disease, can also be caused by superoxide (Sun et al., 2010). As a result, superoxide anion scavenging is critical to antioxidant activity. Our findings show that CSP has a significant scavenging effect on superoxide radicals.

As astable radical, DPPH may be used to test an antioxidant's scavenging capability. As a result, it's been frequently utilized to assess the radical-scavenging abilities of naturally occurring chemicals (Leong and Shui, 2002). Figure 4 depicts the DPPH radical scavenging effects of CSP. In a concentration-dependent 
TABLE 3 | Effects of CSP on the activities of SOD, GSH-Px, TAOC and MDA levels in serums in aging mice.

\begin{tabular}{|c|c|c|c|c|}
\hline Test Groups & SOD (U/mL) & GSH-Px (U/mL) & TAOC (U/mL) & MDA (nmol/ml) \\
\hline Normal control & $277.82 \pm 24.56$ & $158.33 \pm 16.68$ & $16.52 \pm 1.62$ & $11.47 \pm 0.78$ \\
\hline Aging control & $194.33 \pm 17.16^{d}$ & $144.75 \pm 11.52^{\mathrm{c}}$ & $9.69 \pm 1.68^{d}$ & $15.78 \pm 1.06^{d}$ \\
\hline Ascorbic acid (100 mg/kg positive control) & $266.98 \pm 24.53^{b}$ & $177.83 \pm 12.35^{\mathrm{b}}$ & $15.13 \pm 0.34^{b}$ & $11.14 \pm 0.85^{\mathrm{b}}$ \\
\hline $\mathrm{CSP}(100 \mathrm{mg} / \mathrm{kg})$ & $232.47 \pm 17.96^{b}$ & $146.57 \pm 8.69$ & $10.25 \pm 0.82$ & $13.12 \pm 1.05^{b}$ \\
\hline CSP (200 mg/kg) & $257.38 \pm 21.49^{b}$ & $157.68 \pm 11.63^{\mathrm{a}}$ & $12.46 \pm 1.74^{\mathrm{a}}$ & $12.88 \pm 0.97^{b}$ \\
\hline CSP (400 mg/kg) & $276.86 \pm 17.68^{b}$ & $161.32 \pm 13.51^{\mathrm{a}}$ & $15.57 \pm 2.15^{\mathrm{b}}$ & $12.79 \pm 1.15^{\mathrm{b}}$ \\
\hline
\end{tabular}

Values are mean $\pm S D$.

${ }^{a} \mathrm{p}<0.05$.

${ }^{b} \mathrm{p}<0.01$ vs Aging control.

${ }^{c} \mathrm{p}<0.05$.

${ }^{d} \mathrm{p}<0.01$ vs Normal control.

TABLE 4 | Effects of CSP on the activities of SOD, GSH-Px, TAOC and MDA levels in livers in aging mice.

\begin{tabular}{|c|c|c|c|c|}
\hline Groups & SOD (U/mg protein) & GSH-Px (U/mg protein) & TAOC (U/mg protein) & MDA (nmol/mg protein) \\
\hline Normal control & $354.65 \pm 28.56$ & $141.25 \pm 17.35$ & $6.34 \pm 0.14$ & $2.67 \pm 0.24$ \\
\hline Aging control & $188.68 \pm 24.59^{\mathrm{C}}$ & $87.69 \pm 11.72^{\mathrm{C}}$ & $3.24 \pm 0.25^{\mathrm{c}}$ & $5.75 \pm 0.33^{\mathrm{c}}$ \\
\hline Ascorbic acid (100 mg/kg positive control) & $377.35 \pm 31.25^{b}$ & $149.47 \pm 10.56^{b}$ & $5.27 \pm 0.24^{b}$ & $2.94 \pm 0.24^{b}$ \\
\hline CSP (100 mg/kg) & $261.25 \pm 25.69^{b}$ & $98.47 \pm 8.52^{\mathrm{a}}$ & $3.95 \pm 0.24^{a}$ & $4.27 \pm 0.31^{b}$ \\
\hline CSP (200 mg/kg) & $323.25 \pm 25.17^{\mathrm{b}}$ & $124.54 \pm 9.65^{\mathrm{b}}$ & $4.73 \pm 0.18^{b}$ & $4.01 \pm 0.36^{b}$ \\
\hline CSP (400 mg/kg) & $373.14 \pm 19.35^{b}$ & $147.25 \pm 15.38^{b}$ & $5.74 \pm 0.37^{b}$ & $3.76 \pm 0.32^{b}$ \\
\hline
\end{tabular}

Values are mean $\pm S D$.

${ }^{a} \mathrm{p}<0.05$.

${ }_{\mathrm{p}}^{\mathrm{p}}<0.01$ vs Aging control.

${ }^{c} \mathrm{p}<0.01$ vs Normal control.

way, CSP scavenged the DPPH radical. Within the test dose range, CSP and ascorbic acid had their highest scavenging activities at 3,750 and $250 \mathrm{mg} / \mathrm{L}$, respectively. CSP's scavenging ability against DPPH radical had an $\mathrm{EC}_{50}$ of $603.2 \mathrm{mg} / \mathrm{L}$, which was greater than ascorbic acid's $(34.9 \mathrm{mg} / \mathrm{L})$. The typical absorption maxima of DPPH in alcohol is $517 \mathrm{~nm}$. When a hydrogen-atom-donating antioxidant is introduced to $\mathrm{DPPH}$, the DPPH radical is scavenged, and the absorbance at $517 \mathrm{~nm}$ vanishes (Marinova and Yanishlieva, 1997). These findings suggest that CSP scavenges DPPH by acting as an electron or hydrogen donor.

A compound's reducing capacity is an important indication of its potential antioxidant action (Kallithraka et al., 2001). The FRAP of CSP rose with the rise in concentration, but stayed low in comparison to ascorbic acid, as seen in Figure 5. The existence of reductones, which serve as antioxidants by disintegrating the freeradical chain after a donation of a hydrogen atom, was typically linked to the reducing capabilities (Amarowicz et al., 2004). The antioxidant activity of a substance can be determined by its reducing capacity (Meir et al., 1995). The current findings on CSP's FRAP suggest that it has an impact on its antioxidant activity.

\section{Antioxidant Activity of CSP in vivo}

Tables 3 and 4 demonstrate the influence of CSP on SOD, CAT, GSH-Px, TAOC, and MDA in the sera and livers of aged mice. As opposed to the normal control group, the activities of antioxidant enzymes (SOD and GSH-Px) and the amount of TAOC in the sera and livers of the aging control group substantially reduced $(p<0.01$; GSH-Px in livers $p<0.05)$, whereas MDA underwent a significant increase $(p<0.01)$. These findings suggest that the aging mice model was successfully developed. In all dosages examined, CSP considerably enhanced $(p<0.01)$ SOD activity and significantly lowered $(p<0.01)$ MDA levels as opposed to the aging control. The extract significantly enhanced $(p<0.01) \mathrm{GSH}-$ Px activity and TAOC level in a dose-dependent fashion.

Antioxidant enzymes are biological macromolecules initial line of defense against oxidative damage. SOD and GSH-Px are key antioxidant defense systems enzymatically (Zhang et al., 2003). SOD, by catalyzing superoxide elimination, defends against oxygen free radicals, thereby damaging membranes and biological entities, whereas GSH-Px catalyzes the reduction of $\mathrm{H}_{2} \mathrm{O}_{2}$ to $\mathrm{O}_{2}$ and $\mathrm{H}_{2} \mathrm{O}$ at the expense of $\mathrm{GSH}$ ( $\mathrm{Lv}$ et al., 2007). The non-enzymatic antioxidant defense mechanism in animals can be reflected in the amount of TAOC (Zhang et al., 2003). In the current study, CSP enhanced TAOC and elevated SOD and GSH-Px activity in aged mice. The findings suggest that CSP's antioxidative mechanism is partially due to its effects on antioxidant enzymes as well as non-enzymatic systems.

As a primary product of lipid peroxidation, MDA, functions as an oxidative damage indicator and aging assessment index (Shen et al., 2018). It's one of the most common indicators of endogenous lipid peroxidation. MDA production rises with age, indicating that peroxidative damage rises as well (Rotruck et al., 1973). Because of the reduction in MDA generation in aged mice, our findings suggest that CSP was effective in inhibiting 
lipid peroxidation. Earlier investigations (Zhong et al., 2013; Govindan et al., 2016) have also depicted that polysaccharides significantly lower MDA levels when compared against the control group.

\section{CONCLUSION}

The individual and interactive impact of process variables (temperature, liquid-to-solid ratio, and per extraction time) on CSP yield were effectively optimized and evaluated using RSM. The findings revealed that process factors had a substantial impact on CSP maximum yield. The experimental values for the polysaccharide yield were quite near to the predicted yield under these optimized circumstances. In vitro antioxidant activities of hydroxyl radicals, superoxide anion radicals, $\mathrm{DPPH}$, and FRAP revealed that CSP possesses antioxidant properties that increase with increasing polysaccharide content. CSP substantially incremented the activity of antioxidant enzymes and dramatically decreased the concentration of MDA within the liver and serum of aging mice in anti-aging tests. CSP substantially reduces agingrelated damage by replenishing antioxidant enzymes and lowering lipid peroxide, according to the study. As a result, CSP demonstrates promising potential to be incorporated into the diet as a functional natural food to prevent and treat agingrelated damage.

\section{REFERENCES}

Amarowicz, R., Pegg, R. B., Rahimi-Moghaddam, P., Barl, B., and Weil, J. A. (2004). Free-Radical Scavenging Capacity and Antioxidant Activity of Selected Plant Species from the Canadian Prairies. Food Chem. 84, 551-562. doi:10.1016/ S0308-8146(03)00278-4

Bezerra, M. A., Santelli, R. E., Oliveira, E. P., Villar, L. S., and Escaleira, L. A. (2008). Response Surface Methodology (RSM) as a Tool for Optimization in Analytical Chemistry. Talanta 76, 965-977. doi:10.1016/j.talanta.2008.05.019

Chaiittianan, R., Chayopas, P., Rattanathongkom, A., Tippayawat, P., and Sutthanut, K. (2016). Anti-Obesity Potential of Corn Silks: Relationships of Phytochemicals and Antioxidation, Anti-Pre-Adipocyte Proliferation, AntiAdipogenesis, and Lipolysis Induction. J. Funct. Foods 23, 497-510. doi:10.1016/j.jff.2016.03.010

Chaiklahan, R., Chirasuwan, N., Triratana, P., Loha, V., Tia, S., and Bunnag, B. (2013). Polysaccharide Extraction from Spirulina Sp. And its Antioxidant Capacity. Int. J. Biol. Macromol. 58, 73-78. doi:10.1016/j.ijbiomac.2013.03.046

Chen, S., Chen, H., Tian, J., Wang, J., Wang, Y., and Xing, L. (2014). EnzymolysisUltrasonic Assisted Extraction, Chemical Characteristics and Bioactivities of Polysaccharides from Corn Silk. Carbohydr. Polym. 101, 332-341. doi:10.1016/ j.carbpol.2013.09.046

Chen, S., Chen, H., Tian, J., Wang, Y., Xing, L., and Wang, J. (2013). Chemical Modification, Antioxidant and a-amylase Inhibitory Activities of Corn Silk Polysaccharides. Carbohydr. Polym. 98, 428-437. doi:10.1016/ j.carbpol.2013.06.011

Das, K., and Roychoudhury, A. (2014). Reactive Oxygen Species (ROS) and Response of Antioxidants as ROS-Scavengers During Environmental Stress in Plants. Front. Environ. Sci. 2, 53. doi:10.3389/fenvs.2014.00053

Dubois, M., Gilles, K. A., Hamilton, J. K., Rebers, P. A., and Smith, F. (1956). Colorimetric Method for Determination of Sugars and Related Substances. Anal. Chem. 28, 350-356. doi:10.1021/ac60111a017

Gao, C., Wang, Y., Wang, C., and Wang, Z. (2013). Antioxidant and Immunological Activity In Vitro of Polysaccharides from Gomphidius

\section{DATA AVAILABILITY STATEMENT}

The raw data supporting the conclusions of this article will be made available by the authors, without undue reservation.

\section{ETHICS STATEMENT}

The animal study was reviewed and approved by The animal experiment was reviewed and approved by the Ethics Committee from the Experimental Animal Center of Jilin University (Changchun, China). The production license number is SCXK (Liao) 2020-001.

\section{AUTHOR CONTRIBUTIONS}

LZ and YY conducted the experiment and analyzed the data. LZ and ZW designed the study and wrote the manuscript. ZW have funding for the work.

\section{FUNDING}

This work was supported by Liaoning Revitalization Talents Program (Grant No. XLYC1807034) and the Talent Program of Shenyang Agricultural University (Grant No. 2021Y001).

rutilus Mycelium. Carbohydr. Polym. 92, 2187-2192. doi:10.1016/ j.carbpol.2012.12.011

Ge, Q., Mao, J.-W., Zhang, A.-Q., Wang, Y.-J., and Sun, P.-L. (2013). Purification, Chemical Characterization, and Antioxidant Activity of a Polysaccharide from the Fruiting Bodies of Sanghuang Mushroom (Phellinus Baumii Pilát). Food Sci. Biotechnol. 22, 301-307. doi:10.1007/s10068-013-0081-1

Govindan, S., Johnson, E. E., Christopher, J., Shanmugam, J., Thirumalairaj, V., and Gopalan, J. (2016). Antioxidant and Anti-Aging Activities of Polysaccharides from Calocybe Indica Var. APK2. Exp. Toxicol. Pathol. 68, 329-334. doi:10.1016/j.etp.2016.04.001

Hasanudin, K., Hashim, P., and Mustafa, S. (2012). Corn Silk (Stigma Maydis) in Healthcare: A Phytochemical and Pharmacological Review. Molecules 17, 9697-9715. doi:10.3390/molecules17089697

Kallithraka, S., Bakker, J., Clifford, M. N., and Vallis, L. (2001). Correlations between Saliva Protein Composition and Some T-I Parameters of Astringency. Food Qual. Preference 12, 145-152. doi:10.1016/S0950-3293(00)00040-9

Leong, L. P., and Shui, G. (2002). An Investigation of Antioxidant Capacity of Fruits in Singapore Markets. Food Chem. 76, 69-75. doi:10.1016/S03088146(01)00251-5

Li, X., Wang, L., and Wang, B. (2017). Optimization of Encapsulation Efficiency and Average Particle Size of Hohenbuehelia Serotina Polysaccharides Nanoemulsions Using Response Surface Methodology. Food Chem. 229, 479-486. doi:10.1016/j.foodchem.2017.02.051

Li, X., Wang, Z., Wang, L., Walid, E., and Zhang, H. (2012). In Vitro Antioxidant and Anti-proliferation Activities of Polysaccharides from Various Extracts of Different Mushrooms. Int. J. Mol. Sci. 13, 5801-5817. doi:10.3390/ ijms13055801

Liu, J., Wang, C., Wang, Z., Zhang, C., Lu, S., and Liu, J. (2011). The Antioxidant and Free-Radical Scavenging Activities of Extract and Fractions from Corn Silk (Zea mays L.) and Related Flavone Glycosides. Food Chem. 126, 261-269. doi:10.1016/j.foodchem.2010.11.014

Lv, L., Gu, X., Tang, J., and Ho, C. (2007). Antioxidant Activity of Stilbene Glycoside from Polygonum Multiflorum Thunb In Vivo. Food Chem. 104, 1678-1681. doi:10.1016/j.foodchem.2007.03.022 
Marinova, E. M., and Yanishlieva, N. V. (1997). Antioxidative Activity of Extracts from Selected Species of the Family Lamiaceae in sunflower Oil. Food Chem. 58, 245-248. doi:10.1016/S0308-8146(96)00223-3

Meir, S., Kanner, J., Akiri, B., and Philosoph-Hadas, S. (1995). Determination and Involvement of Aqueous Reducing Compounds in Oxidative Defense Systems of Various Senescing Leaves. J. Agric. Food Chem. 43, 1813-1819. doi:10.1021/jf00055a012

Necyk, C., and Zubach-Cassano, L. (2017). Natural Health Products and Diabetes: A Practical Review. Can. J. Diabetes 41, 642-647. doi:10.1016/j.jcjd.2017.06.014

Pan, Y., Wang, C., Chen, Z., Li, W., Yuan, G., and Chen, H. (2017). Physicochemical Properties and Antidiabetic Effects of a Polysaccharide from Corn Silk in High-Fat Diet and Streptozotocin-Induced Diabetic Mice. Carbohydr. Polym. 164, 370-378. doi:10.1016/j.carbpol.2017.01.092

Qi, H., Zhang, Q., Zhao, T., Chen, R., Zhang, H., Niu, X., et al. (2005). Antioxidant Activity of Different Sulfate Content Derivatives of Polysaccharide Extracted from Ulva Pertusa (Chlorophyta) In Vitro. Int. J. Biol. Macromol. 37, 195-199. doi:10.1016/j.ijbiomac.2005.10.008

Raza, A., Li, F., Xu, X., and Tang, J. (2017). Optimization of Ultrasonic-Assisted Extraction of Antioxidant Polysaccharides from the Stem of Trapa Quadrispinosa Using Response Surface Methodology. Int. J. Biol. Macromol. 94, 335-344. doi:10.1016/j.ijbiomac.2016.10.033

Rotruck, J. T., Pope, A. L., Ganther, H. E., Swanson, A. B., Hafeman, D. G., and Hoekstra, W. G. (1973). Selenium: Biochemical Role as a Component of Glutathione Peroxidase. Science 179, 588-590. doi:10.1126/science.179.4073.588

Shah, M. A., Bosco, S. J., and Mir, S. A. (2014). Plant Extracts as Natural Antioxidants in Meat and Meat Products. Meat Sci. 98, 21-33. doi:10.1016/ j.meatsci.2014.03.020

Shen, S., Xu, Z., Feng, S., Wang, H., Liu, J., Zhou, L., et al. (2018). Structural Elucidation and Antiaging Activity of Polysaccharide from Paris Polyphylla Leaves. Int. J. Biol. Macromol. 107, 1613-1619. doi:10.1016/ j.ijbiomac.2017.10.026

Shi, M., Zhang, Z., and Yang, Y. (2013). Antioxidant and Immunoregulatory Activity of Ganoderma Lucidum Polysaccharide (GLP). Carbohydr. Polym. 95, 200-206. doi:10.1016/j.carbpol.2013.02.081

Smirnoff, N., and Cumbes, Q. J. (1989). Hydroxyl Radical Scavenging Activity of Compatible Solutes. Phytochemistry 28, 1057-1060. doi:10.1016/0031-9422(89) 80182-7

Sun, Z.-W., Zhang, L.-X., Zhang, B., and Niu, T.-G. (2010). Structural Characterisation and Antioxidant Properties of Polysaccharides from the Fruiting Bodies of Russula Virescens. Food Chem. 118, 675-680. doi:10.1016/j.foodchem.2009.05.036

Tian, S., Hao, C., Xu, G., Yang, J., and Sun, R. (2017). Optimization Conditions for Extracting Polysaccharide from Angelica Sinensis and its Antioxidant Activities. J. Food Drug Anal. 25, 766-775. doi:10.1016/j.jfda.2016.08.012

Ueda, J., Saito, N., Shimazu, Y., and Ozawa, T. (1996). A Comparison of Scavenging Abilities of Antioxidants against Hydroxyl Radicals. Arch. Biochem. Biophys. 333, 377-384. doi:10.1006/abbi.1996.0404
Wang, Z., Zhao, Y., and Su, T. (2015). Extraction and Antioxidant Activity of Polysaccharides from Rana chensinensis Skin. Carbohydr. Polym. 115, 25-31. doi:10.1016/j.carbpol.2014.08.082

Wu, Y., Yi, L., Li, E., Li, Y., Lu, Y., Wang, P., et al. (2017). Optimization of Glycyrrhiza Polysaccharide Liposome by Response Surface Methodology and its Immune Activities. Int. J. Biol. Macromol. 102, 68-75. doi:10.1016/ j.ijbiomac.2017.04.006

Yang, J., Li, X., Xue, Y., Wang, N., and Liu, W. (2014). Anti-Hepatoma Activity and Mechanism of Corn Silk Polysaccharides in H22 Tumor-Bearing Mice. Int. J. Biol. Macromol. 64, 276-280. doi:10.1016/j.ijbiomac.2013.11.033

Yildirim, A., Mavi, A., and Kara, A. A. (2001). Determination of Antioxidant and Antimicrobial Activities of Rumex Crispus L. Extracts. J. Agric. Food Chem. 49, 4083-4089. doi:10.1021/jf0103572

Yu, Y., Shen, M., Song, Q., and Xie, J. (2018). Biological Activities and Pharmaceutical Applications of Polysaccharide from Natural Resources: A Review. Carbohydr. Polym. 183, 91-101. doi:10.1016/j.carbpol.2017.12.009

Zhang, Q., Li, N., Zhou, G., Lu, X., Xu, Z., and Li, Z. (2003). In Vivo Antioxidant Activity of Polysaccharide Fraction from Porphyra Haitanesis (Rhodephyta) in Aging Mice. Pharmacol. Res. 48, 151-155. doi:10.1016/S1043-6618(03)00103-8

Zhao, H. P., Zhang, Y., Liu, Z., Chen, J. Y., Zhang, S. Y., Yang, X. D., et al. (2017). Acute Toxicity and Anti-Fatigue Activity of Polysaccharide-Rich Extract from Corn Silk. Biomed. Pharmacother. 90, 686-693. doi:10.1016/ j.biopha.2017.04.045

Zhao, W., Yin, Y., Yu, Z., Liu, J., and Chen, F. (2012). Comparison of Anti-Diabetic Effects of Polysaccharides from Corn Silk on normal and Hyperglycemia Rats. Int. J. Biol. Macromol 50, 1133-1137. doi:10.1016/j.ijbiomac.2012.02.004

Zhong, W., Liu, N., Xie, Y., Zhao, Y., Song, X., and Zhong, W. (2013). Antioxidant and Anti-Aging Activities of Mycelial Polysaccharides from Lepista Sordida. Int. J. Biol. Macromol. 60, 355-359. doi:10.1016/j.ijbiomac.2013.06.018

Conflict of Interest: The authors declare that the research was conducted in the absence of any commercial or financial relationships that could be construed as a potential conflict of interest.

Publisher's Note: All claims expressed in this article are solely those of the authors and do not necessarily represent those of their affiliated organizations, or those of the publisher, the editors and the reviewers. Any product that may be evaluated in this article, or claim that may be made by its manufacturer, is not guaranteed or endorsed by the publisher.

Copyright (c) 2021 Zhang, Yang and Wang. This is an open-access article distributed under the terms of the Creative Commons Attribution License (CC BY). The use, distribution or reproduction in other forums is permitted, provided the original author(s) and the copyright owner(s) are credited and that the original publication in this journal is cited, in accordance with accepted academic practice. No use, distribution or reproduction is permitted which does not comply with these terms. 